Test Method

\title{
Research on the water blade electrode method for assessing water tree resistance of cross-linked polyethylene
}

\author{
Chengcheng Zhang ${ }^{\mathrm{a}, \mathrm{b},{ }^{*}}$, Chunyang $\mathrm{Li}^{\mathrm{a}}$, Linlin Nie ${ }^{\mathrm{a}}$, Ziang Jing ${ }^{\mathrm{a}}$, \\ Baozhong Han ${ }^{\text {a, c,* }}$ \\ ${ }^{a}$ Key Laboratory of Engineering Dielectrics and Its Application, Ministry of Education, Harbin \\ University of Science and Technology, Harbin 150080, P R China \\ ${ }^{b}$ Yuancheng Cable Co., Ltd., Yixing 214200, P R China \\ 'Shanghai Qifan Wire and Cable Co., Ltd., Shanghai 200008, P R China \\ Corresponding author: e-mail cczh0111@126.com; hbzhlj@163.com
}

Phone: $+86-451-86391657$

\begin{abstract}
A new water blade electrode method is presented to accelerate water tree initiation and propagation in cross-linked polyethylene (XLPE) insulation, and the validity was verified by a series of experiments. Water tree morphology and length over time were monitored using a polarized light microscope and compared to the results through the conventional water needle electrode method. The influence of electric field distribution along the water electrodes on water tree initiation and morphology was also investigated on the basis of experimental data and finite element simulation. Furthermore, the effect of voltage frequency on water tree initiation was also investigated. It was found that the water blade electrode method could greatly accelerate water tree initiation and propagation and it was more convenient to slice XLPE for observation. The high frequency voltage could more efficiently facilitate water tree initiation and propagation.
\end{abstract}

Keywords: XLPE; Water tree; Water blade electrode; Finite element simulation 


\section{Introduction}

Under the combined effects of moisture and electric field, the initiation and propagation of water trees is one of the main aging phenomena in middle and low voltage cross-linked polyethylene (XLPE) insulated underground power cable $[1,2]$. Once water trees form, they will gradually transform to electrical trees as the electric field on the edge of water trees continuously concentrates. Electrical trees can lead to breakdown of cable insulation in a short period of time, and result in power supply blackout [3]. The initiation and propagation of water trees shorten the service life of power cable, and become a potential threat to the power supply security of networks. Therefore, the development and application of water tree retardant XLPE (WTR-XLPE) insulation materials have important theoretical and practical significance in improving the quality of cable insulation.

Many routines have been developed to inhibit the initiation and propagation of water trees, and a series of WTR-XLPE insulation materials have been prepared [4-8]. In actual operation, water tree aging of XLPE insulation materials is a very slow process, and may take a couple of years or decades. Therefore, accelerated aging tests are adopted by most of the cable manufacturers and users to assess the performance of WTR-XLPE insulation materials [9]. The most common method is by creating defects using a water needle electrode which can provide local high electric field and water for water tree initiation and directly cultivate water trees. Then, the specimens are sliced and observed using a polarized light microscope for measuring the lengths of water trees. However, the water needle electrode method has a complicated preparation procedure and the number of initiation positions for water trees in one specimen is limited. At the same time, it is difficult to slice the specimen for 
observing water trees, and the slicing position easily deviates leading to the dispersion of experimental data. Therefore, it is emphasized as an important subject in the insulation materials and cable industry to search for a fast and accurate method for assessing water tree resistance of XLPE insulation materials.

In this paper, a new water blade electrode method is presented to accelerate the initiation and propagation of water trees based on the original water needle electrode method. It was verified through a series of experiments and finite element simulation results of electric field distribution along the water electrode that using the water blade electrode could improve the initiation rate of water trees, facilitate slicing for observing water trees and reduce the dispersion of experimental data. The water blade electrode method can assess water tree resistance of XLPE insulation materials more efficiently and accurately. Water tree initiation and propagation in XLPE and WTR-XLPE insulation materials under different voltage frequencies were also studied using the water blade electrode method.

\section{Experimental}

\subsection{Materials}

The low density polyethylene (LDPE, LD200BW) pellets with a density of 0.922 $\mathrm{g} / \mathrm{cm}^{3}$ and a Melt Flow Index (MFI) of $2.0 \mathrm{~g} / 10$ min were purchased from Beijing Yanshan Petroleum Co. Ltd., China. Dicumylperoxide (DCP) was obtained from Shanghai Gaoqiao Petroleum Co. Ltd., China. Methylene blue was purchased from Tianjin Zhiyuan Chemical Reagen Co. Ltd., China. Sodium chloride (NaCl) was supplied by Beijing Petsun Chemical Technology Co. Ltd., China. All the above chemicals were used as received without further purification.

\subsection{Specimen preparation}


LDPE and water-tree-retardant cross-linkable PE were compression moulded under a pressure of $15 \mathrm{MPa}$ at $110{ }^{\circ} \mathrm{C}$ and then crosslinked under the same pressure at 175 ${ }^{\circ} \mathrm{C}$ for 30 minutes to obtain the specimens of size $100 \mathrm{~mm} \times 100 \mathrm{~mm} \times 4 \mathrm{~mm}$.

\subsection{Water tree tests}

The specimens for water tree tests were divided into two groups for testing with the water blade and water needle electrode methods, respectively. For the water blade electrode method, the specimen shape and the arrangement of cuts are shown in Fig. 1a. Two cuts were made by using a blade perpendicular to the surface, leaving a residual thickness between the blade and the other surface of the specimen of $2 \mathrm{~mm}$. The two cuts acted as the defects in the insulation and would be the starting point of water tree initiation and propagation. For the water needle electrode method, the specimen shape and the arrangement of pinholes are shown in Fig. 1b. Seven pinholes were obtained by using a needle to prick the specimen perpendicular to the surface, and the residual thickness was also $2 \mathrm{~mm}$. After the defects were introduced, the aluminum electrode was deposited on the other surface of the specimen by a vacuum coating system as the grounding electrode.

Fig. 2 shows the experimental setup for water tree tests. After a PP pipe was glued to the surface of the specimen with defects, a little $1.8 \mathrm{M} \mathrm{NaCl}$ water solution was poured into the PP pipe, and then the pipe and the specimen together with the solution were all kept in vacuum oven for 30 min to remove the air in the cuts and pinholes. For water tree tests, an $\mathrm{AC}$ voltage of $4 \mathrm{kV}$ with frequency of $50 \mathrm{~Hz}$ or $3 \mathrm{kHz}$ was applied to the specimen through a high voltage line immersed in $1.8 \mathrm{M} \mathrm{NaCl}$ water solution used as the electrolyte for 1-7 days at room temperature. After a certain time, the specimens were cut into at least 16 slices with thickness of $180 \mu \mathrm{m}$ to guarantee 
the cuts and pinholes in the middle of the slices. After the slices were stained with methylene blue for $4 \mathrm{~h}$, the water tree morphology and length in each slice were observed and recorded by a polarized light microscope. Water tree length was determined by the average length of the longest branch of water trees along the direction of electric field.

\section{Results and discussion}

The protrusion at the contact surface of the insulating and semiconducting layers in the cables, and the defects such as chemical residues, water droplets, microvoids, etc. in the insulation generally have higher electric field and become water tree inception points. In this paper, the water blade and water needle gaps were adopted to simulate these defects to accelerate the initiation and propagation of water trees to compare and analyze the two experimental methods and estimate water tree resistance of different XLPE insulation materials.

The morphology of water trees in XLPE initiated by the water blade and water needle electrode methods at a voltage of $4 \mathrm{kV}$ and frequency of $3 \mathrm{kHz}$ is shown in Fig. 3 and 4, respectively. The average length of the longest branch of water trees along the direction of the electric field was statistical treated and the plots of the length versus time are given in Fig. 5.

The morphology of water trees in WTR-XLPE initiated by the water blade and water needle electrode methods at a voltage of $4 \mathrm{kV}$ and frequency of $3 \mathrm{kHz}$ is shown in Fig. 6 and 7, respectively. The average length of the longest branch of water trees along the direction of the electric field was statistical treated and the plots of the 
length versus time are given in Fig. 8.

As seen from the above results, when the water blade electrode method was applied, a fan shaped water tree formed at the tip of cuts and it propagated radially down the tip of cuts. However, when the water needle electrode method was applied, a dense water tree developed at the tip of pinholes and surrounding the water electrode, and it propagated along different directions. In the two electrodes, the length of water trees increased gradually as the application time of the voltage increased. In 1-7 days, the length of water trees initiated by the water blade electrode method was larger compared to the water needle electrode method. To analyze the reason for this, the electric field distribution along the two water electrodes at the early stage of water tree initiation was analyzed by finite element simulation. Under the same condition as the experiments, a voltage of $4 \mathrm{kV}$ with a frequency of $3 \mathrm{kHz}$ was applied to the cut and pinhole. The tip radius of the blade and needle were all set to $10 \mu \mathrm{m}$, and the results from finite element simulation are presented in Fig. 9.

As shown in Fig. 9(a), when the water blade electrode was applied, the maximum value of electric field $(20.7 \mathrm{kV} / \mathrm{mm})$ appeared at the tip of the cut. The electric field of a position slightly away from the tip of the cut reduced quickly and was soon below $10 \mathrm{kV} / \mathrm{mm}$. Hence, a water tree was only initiated from the tip of the cut and propagated mainly radially along the direction of the electric field. Fig. 9(b) indicates that, when the water needle electrode was applied, the maximum value of electric field at the tip of the pinhole had reached $135 \mathrm{kV} / \mathrm{mm}$, which is 67.5 times the average electric field $(2 \mathrm{kV} / \mathrm{mm})$ and 6.5 times the maximum electric field of the water blade 
electrode. The electric field at a distance from the tip was still higher than $20 \mathrm{kV} / \mathrm{mm}$. A lot of water tree inception points existed near the tip of the pinhole and a dense water tree appeared around the pinhole. After initiation, the dense water tree with good conductive performance soon formed electric field shielding of the pinhole and led to significant decline of electric field around the pinhole. As a result, the rate of water tree propagation using the water needle electrode was below that using the water blade electrode.

It was also found that, at the same voltage duration, there were minimal differences and high regularity in water tree morphology and length in the slices from different part of the specimen when the water blade electrode method was applied. When water tree tests were performed using the water needle electrode, water tree morphology and size observed varied greatly in the slices from different pinholes of the specimen. Combined with the results of electric field simulation in Fig. 9, the main causes are analyzed as follows: (1) The electric field at the pinhole was considerably larger than that at the cut, so the initiation and propagation of water trees was quite random when the water needle electrode was applied. (2) When the water blade electrode was applied, the length of the cut was much larger than the width and it can be considered that the electric field applied on the specimen was a two dimensional field perpendicular to the cut direction, and the morphology of water trees was affected little by the slice position. When the water needle electrode was applied, the propagation of water trees below the tip of the pinhole was theoretically fastest. However, it was difficult to take a slice at the tip of the pinhole for observation 
without deviation and a small change in the slice location, although it may be only dozens of micrometers, would inevitably lead to a discrepancy of water tree morphology and length observed. Another advantage can be found for the water blade electrode method. A specimen could be cut into multiple slices for observing water trees, which was convenient for data statistics and analysis, and had high experimental efficiency. However, when the water needle electrode method was applied, only one slice could be obtained at each pinhole, the efficiency of water tree initiation was low, and data statistics and analysis was not convenient.

It was reported that the water tree aging effect with electrical trees is more significant compared to without electrical trees [10]. To prove whether an electrical tree existed in the water tree initiation experiment, further experiments were performed:

(1) The blade was maintained in the XLPE specimen and the $\mathrm{NaCl}$ water solution was substituted with silicone oil. The voltage with the same frequency and amplitude as water tree initiation tests was applied to the specimen.

(2) The blade was maintained in the XLPE specimen and $\mathrm{NaCl}$ water solution was not added. The voltage with the same frequency and amplitude as in water tree initiation tests was applied to the specimen.

Fig. 10 showed the microtopography of XLPE around the tip of the blade when the voltage was applied for 6 days in experiment (1) and experiment (2), and demonstrated that an electrical tree had not appeared in the water tree initiation tests. 
This further confirmed the feasibility of the water blade electrode method for evaluating water tree resistance of XLPE insulation materials.

In general, water trees rarely exist in XLPE under DC voltage and tend to form under AC voltage. It is found that the voltage frequency is an important influence to accelerate the initiate and propagation of water trees. In order to select an appropriate initiation frequency, water tree tests were also performed on XLPE and WTR-XLPE under an applied $\mathrm{AC}$ voltage of $4 \mathrm{kV}$ and frequency of $50 \mathrm{~Hz}$ by the water blade electrode method. The average length of water trees was compared to that under a frequency of $3 \mathrm{kHz}$, and the results were presented in Fig. 11.

From Fig. 11, it can be seen that water tree initiation and propagation was accelerated with increase of the frequency, which demonstrates the electro-mechanical mechanism of water tree propagation in cable insulation materials [11]. Hence, in order to improve water tree initiation rate, reduce the test time and enhance the experimental efficiency, the water blade electrode can be chosen to initiate water trees at higher voltage frequency for assessing water tree resistance of cable insulation materials.

\section{Conclusions}

Based on the original water needle electrode method, a new water blade electrode method has been proposed to accelerate water tree aging of XLPE cable insulation materials, and a series of experiments and finite element simulation were carried out to verify the feasibility and superiority of the method. The conclusions are as follows: 
(1) The water blade electrode method can effectively accelerate water tree aging of XLPE cable insulation materials. Water tree initiation and propagation rates are demonstrably superior to that by the water needle electrode method. In the water tree initiation and propagation process, an electrical tree has not been generated.

(2) The difference between the morphology and size of water trees initiated by the water blade electrode method in identical specimen is relatively low. Water trees propagate gradually over time and have high regularity.

(3) The high electric field mainly concentrates at the tip of the water blade electrode, which leads to the conclusion that water trees were only initiated from the tip and presented a fan-like shape.

(4) Water tree initiation and propagation are accelerated with increase of the frequency by the water blade electrode method, which conforms to the electro-mechanical mechanism of water tree aging in XLPE cable insulation materials. Hence, employing the water blade electrode method and selecting an appropriate voltage frequency could improve water tree initiation rate and reduce the test time for assessing water tree resistance performance of XLPE insulation materials. 


\section{Acknowledgements}

This work is supported by National Science Foundation of China (grant No. 51337002) and Natural Science Foundation for Distinguished Young Scholars of Heilongjiang Province (JC201409). 


\section{References}

[1] E.F. Steennis, F.H. Kreuger. Water treeing in polyethylene cables. IEEE Trans Electr Insul 1990; 25 (5): 989-1028.

[2] C. Kim, J. Jang, X.Y. Huang, P.K. Jiang, H. Kim. Finite element analysis of electric field distribution in water treed XLPE cable insulation (1): The influence of geometrical configuration of water electrode for accelerated water treeing test. Polym Test 2007; 26: 482-488.

[3] R. Ross. Inception and propagation mechanisms of water treeing. IEEE Trans Dielectr Electr Insul1998; 5: 660-680.

[4] X.Y. Huang, F. Liu, P.K. Jiang. Effect of nanoparticle surface treatment on morphology, electrical and water treeing behavior of LLDPE composites. IEEE Trans Dielectr Electr Insul 2010; 17(6): 1697-1704.

[5] Z.S. Ma, X.Y. Huang, P.K. Jiang, G.L. Wang. Effect of silane-grafting on water tree resistance of XLPE cable insulation. J Appl Polym Sci 2010; 115(6): $3168-3176$

[6] Z.S. Ma, P.K. Jiang, L.C. Wang, J.S. Yang. Effect of styrene-ethylene-butadiene-styrene and its synergetic effect with ethylene vinyl acetate on the mechanical, thermal, dielectric, and water-treeing behaviors of crosslinked polyethylene. J Appl Polym Sci 2010; 118(4): 2350-2357.

[7] F. Tian, Y.C. Qiao, Y.R. Ni, C.H. Su. Study on inhibition of water tree in XLPE insulation material. Adv Mater Res 2013; 602-604: 668-671.

[8] X.F. Li, X. Liu, X. Man, D.R. Xie, X.L. Cao, X.Q. Wang, H.J. Liu. Influence of 
compatibilizers on the water-tree property of Montmorillonite/Cross-linked polyethylene nanocomposites. IEEE 10th International Conference on the Properties and Applications of Dielectric Materials 2012; 1-4.

[9] S. Pelissou, R. Harp, R. Bristol, J. Densley, C. Fletcher, C. Katz, F. Kuchta, D. Kung, T. Person, M. Smalley, J.T. Smith. A review of possible methods for defining tree retardant crosslinked polyethylene (TRXLPE). IEEE Electr Insul Mag 2008; 24(5): 22-30.

[10] M. Huang, K. Zhou, W.B. Tao, M.L. Yang, D. Yang. Transformation mechanism of electrical tree to water tree in XLPE cables. International Conference on Power System Technology 2014; 1491-1496.

[11] Z.S. Ma, X.Y. Huang, P.K. Jiang. A comparative study of effects of SEBS and EPDM on the water tree resistance of cross-linked polyethylene. Polym Degrad Stab 2010; 95(9): 1943-1949. 


\section{Figure Captions}

Fig. 1 The schematic diagram of the specimen shape and the arrangement of the defects by applying the (a) water blade electrode and (b) water needle electrode

Fig. 2 The schematic diagram of the experimental setup for water tree tests

Fig. 3 The morphology of water tree in XLPE initiated by the water blade electrode method

Fig. 4 The morphology of water tree in XLPE initiated by the water needle electrode method

Fig. 5 The length of water tree in XLPE

Fig. 6 The morphology of water tree in WTR-XLPE initiated by the water blade electrode method

Fig. 7 The morphology of water tree in WTR-XLPE initiated by the water needle electrode method

Fig. 8 The length of water tree in WTR-XLPE

Fig. 9 The electric field distribution along the (a) water blade electrode and (b) water needle electrode

Fig. 10 The microtopography of XLPE around the tip of the blade in the experiment (1) and (2)

Fig. 11 The length of water tree at different frequencies 


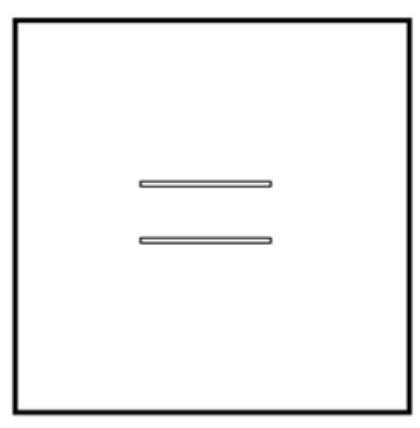

(a)

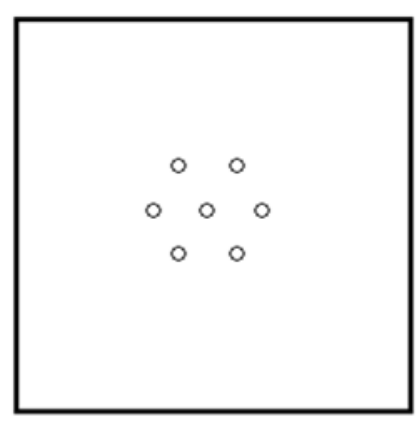

(b)

Fig. 1 


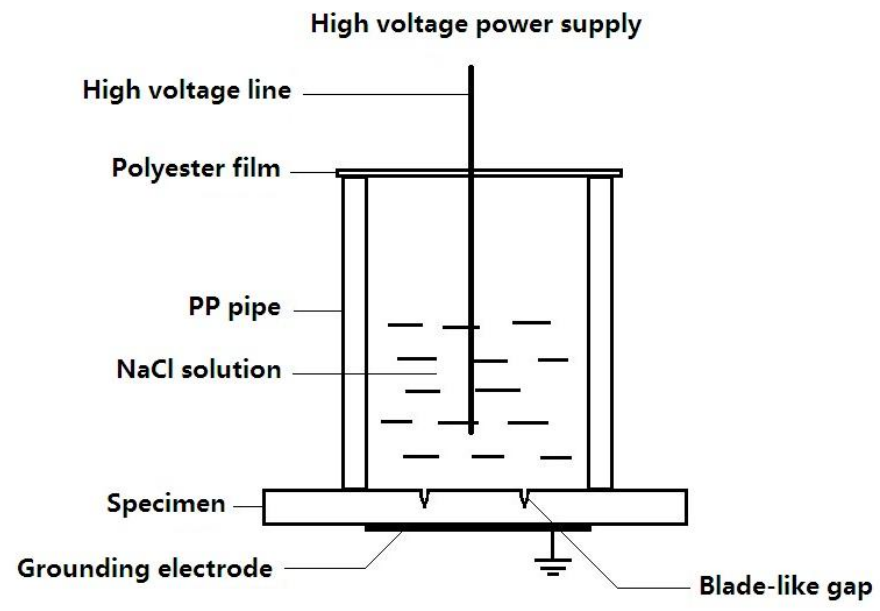

Fig. 2 
Fig. 3 


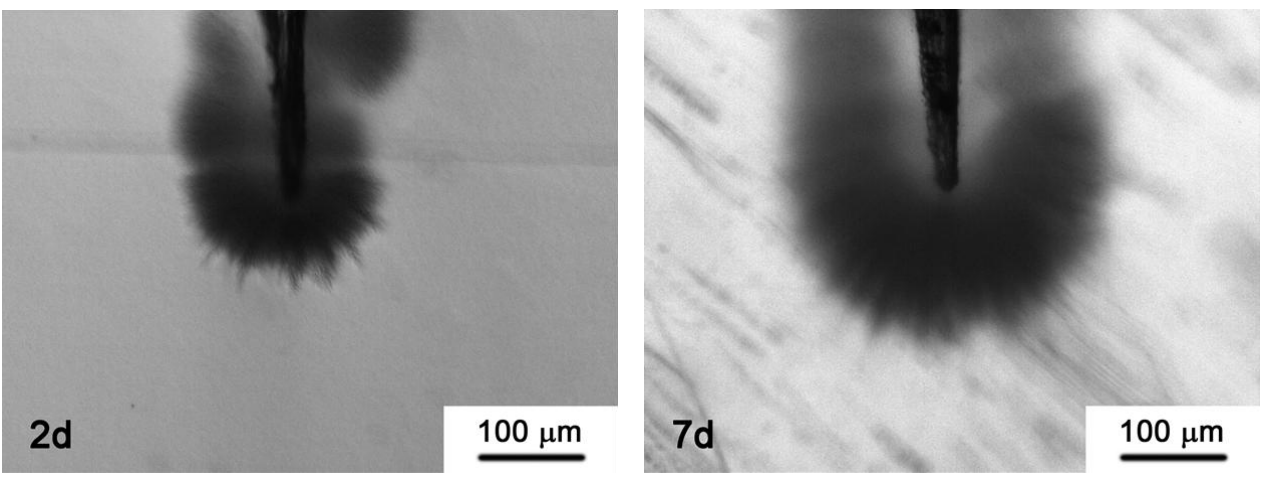

Fig. 4 


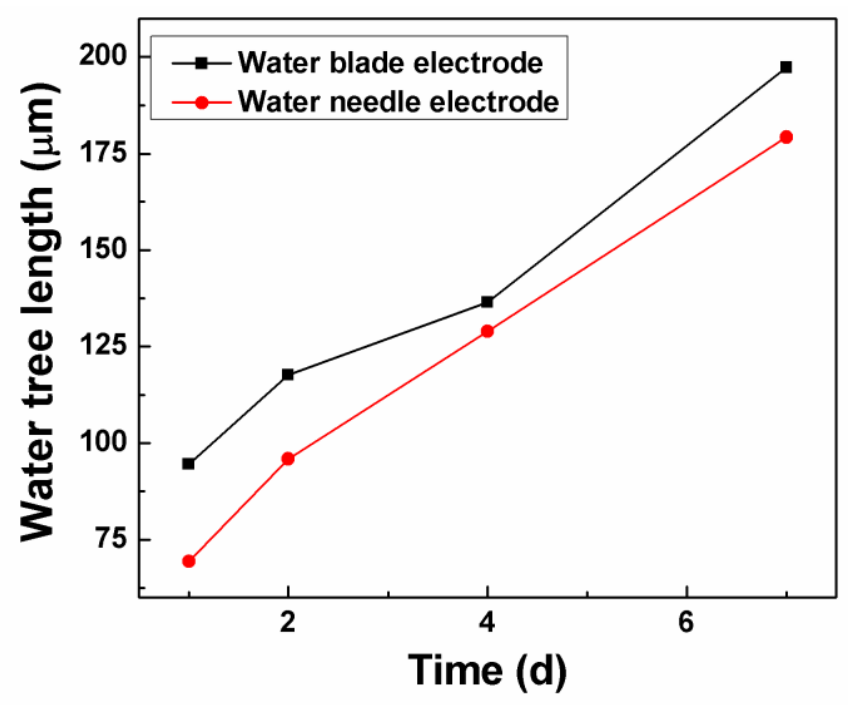

Fig.5 

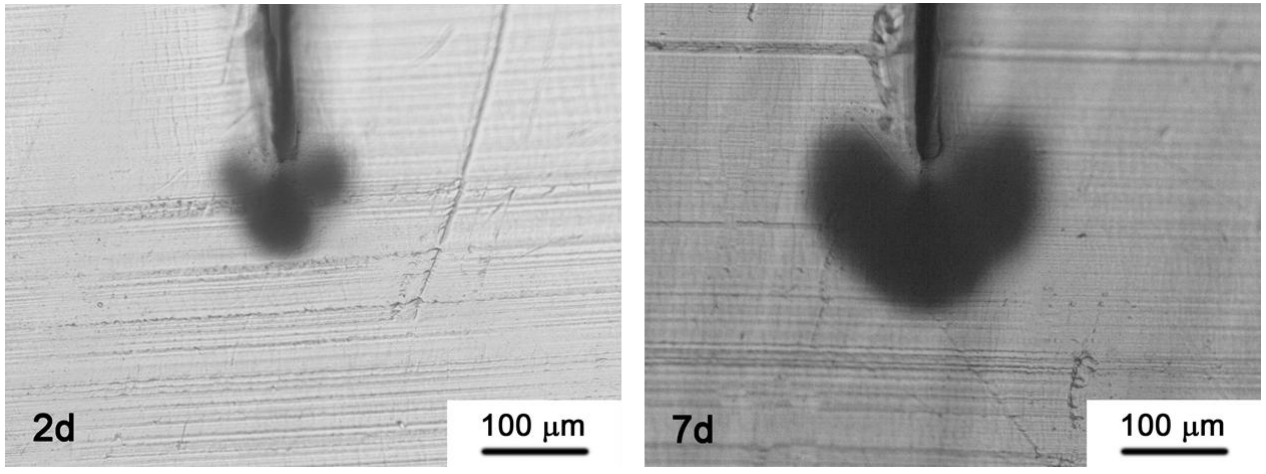

Fig. 6 


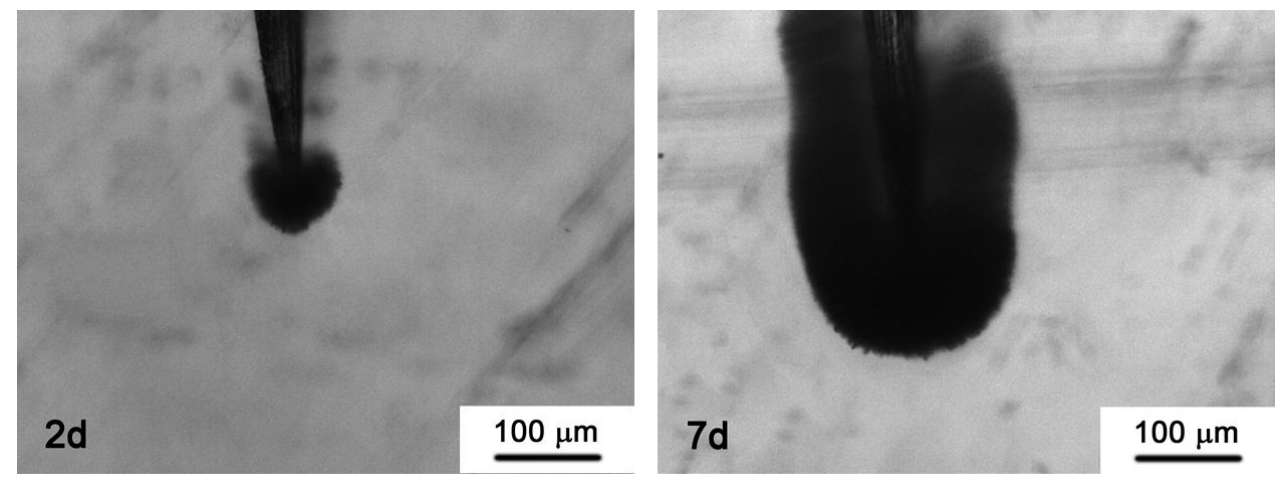

Fig. 7 


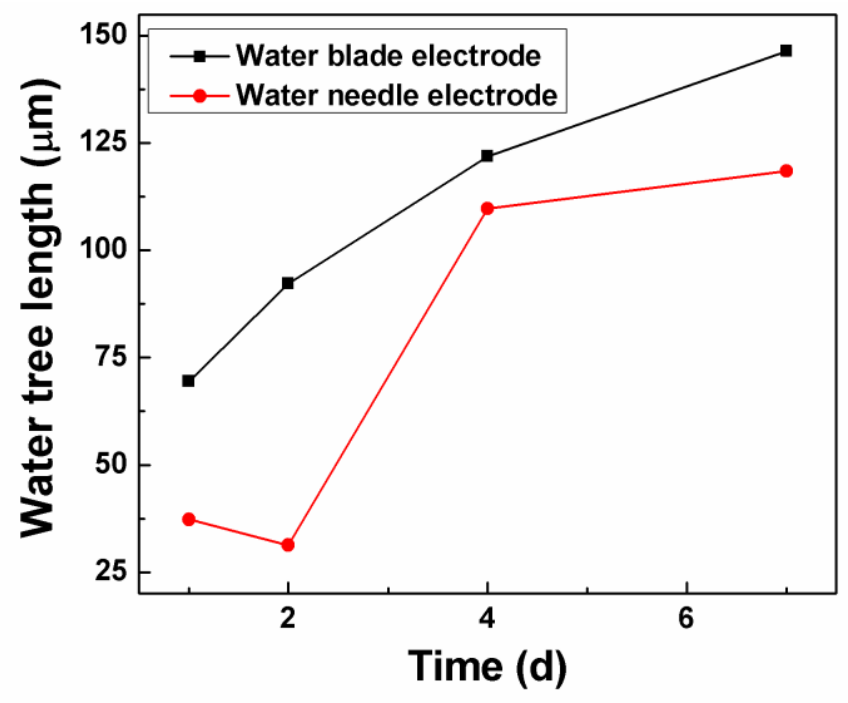

Fig. 8 
(a)

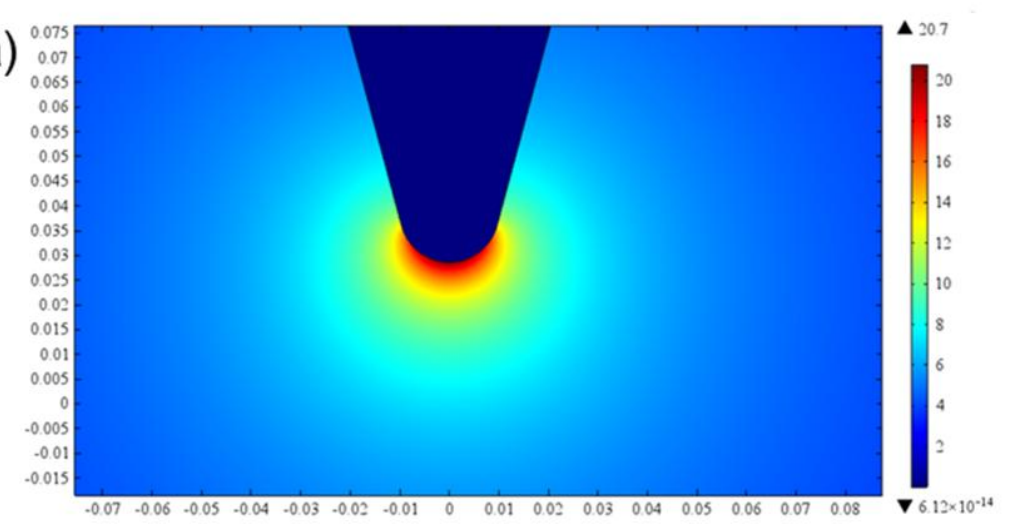

(b)

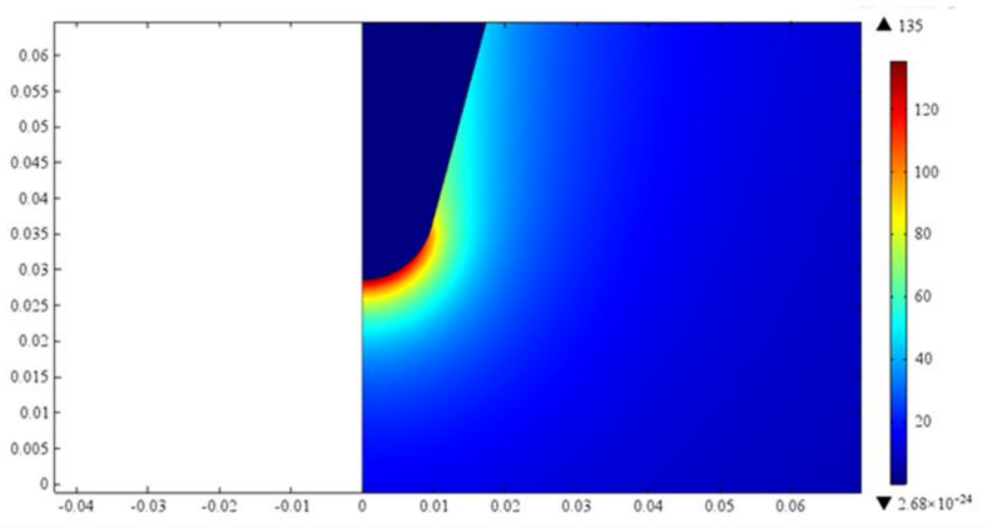

Fig. 9 
(1)

(2)

$100 \mu \mathrm{m}$

$100 \mu \mathrm{m}$

Fig. 10 


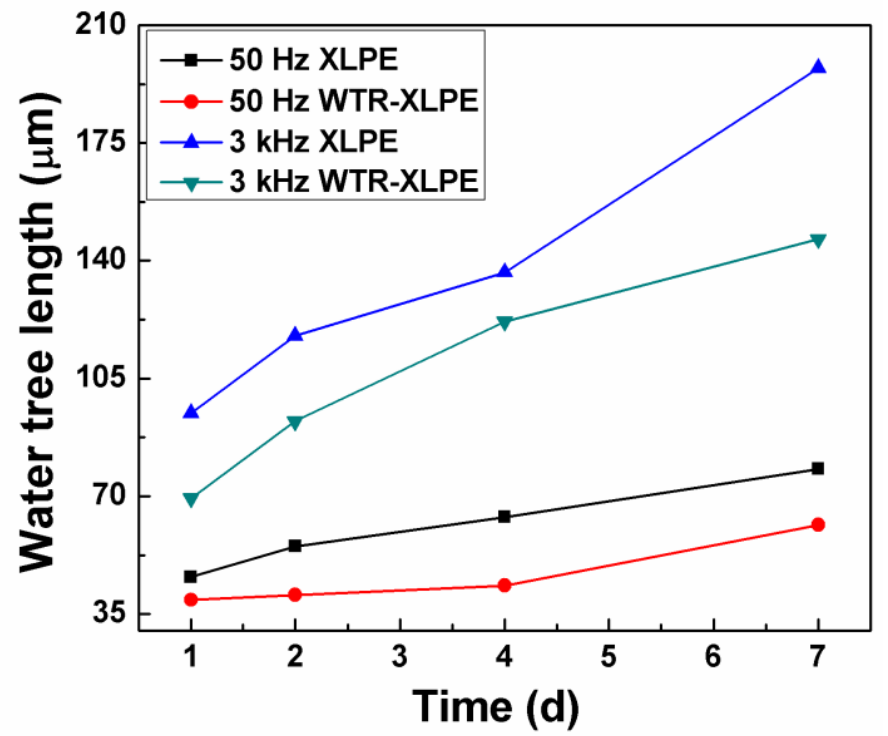

Fig. 11 